\title{
Controlling a large CPU farm using industrial tools
}

Alba Sambade Varela

On behalf of the $\mathrm{LHCb}$ Online group

Real Time 2009

May 10-15 IHEP Beijing 


\section{ECS and data flow in $\mathrm{LHCb}$}

High Level Trigger Farm

- Second levelıof trigger

- input rate: Trigger

- input rate: $1 \mathrm{MH}$

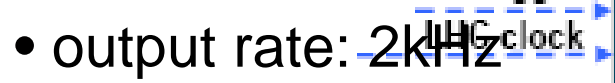

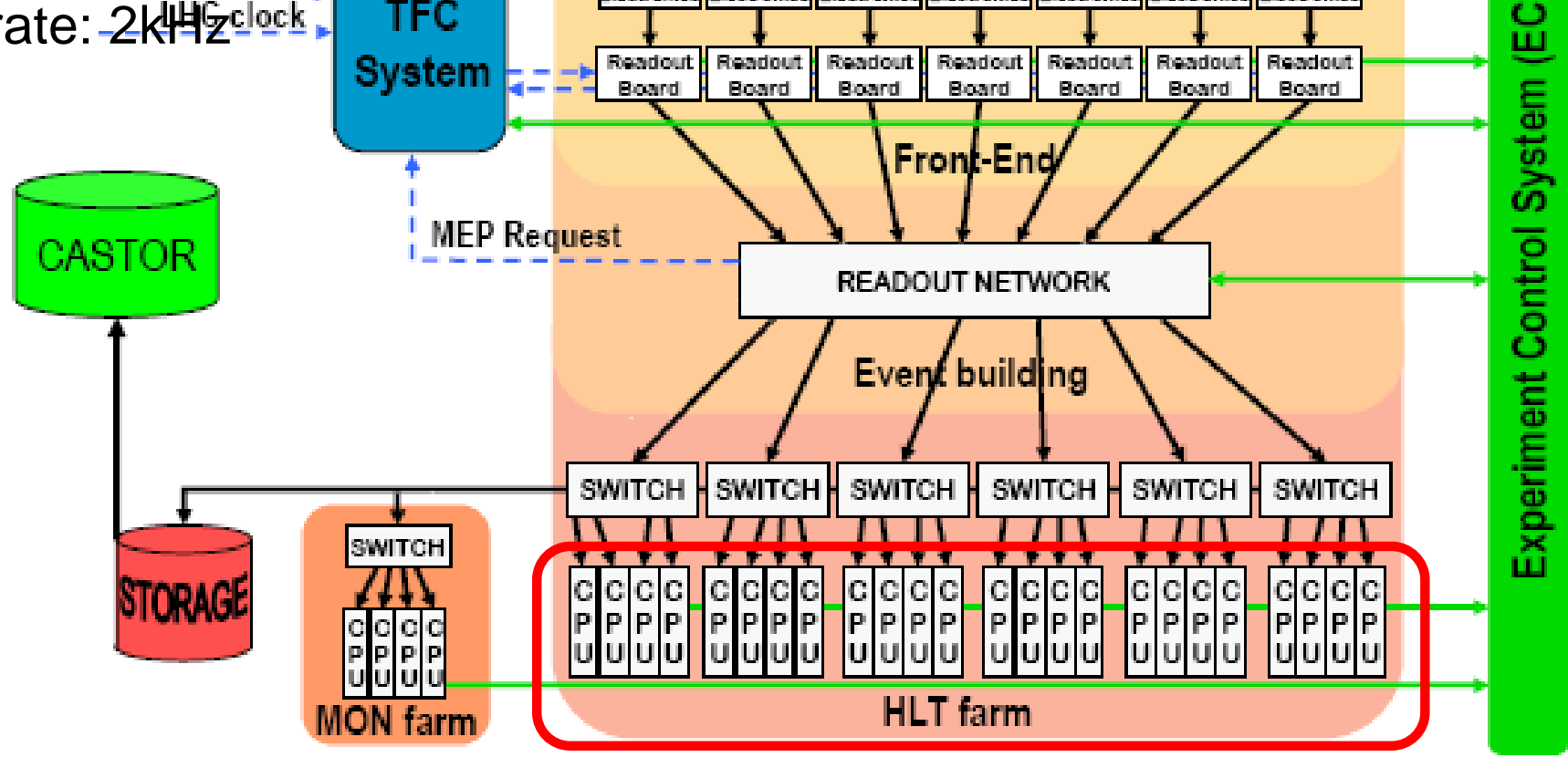

\section{onturar}

Event data

- - Timing and Fast Control Signals

Control and Monitoring data

RTSA2-3: A 40MHz Trigger-Free Readout Architecture

for the LHCb Experiment at CERN
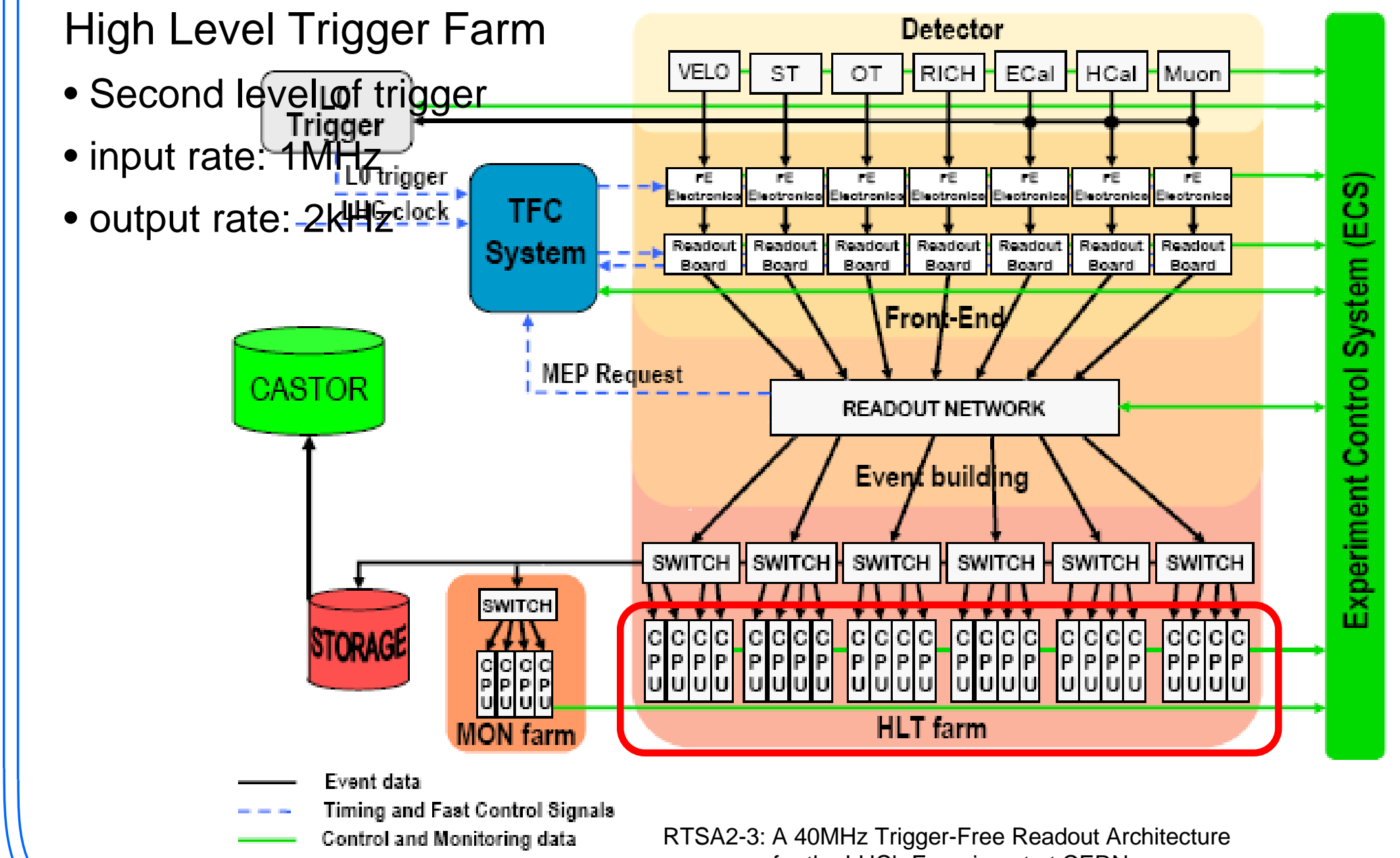


\section{Experiment Control System}

- ECS is in charge of the configuration, operation and supervision of all the online components in LHCb.

- Industrial SCADA system: PVSS

- FSM package

- Definition in terms of hierarchies of Finite State Machines.

- Distributed Information Management System (DIM)

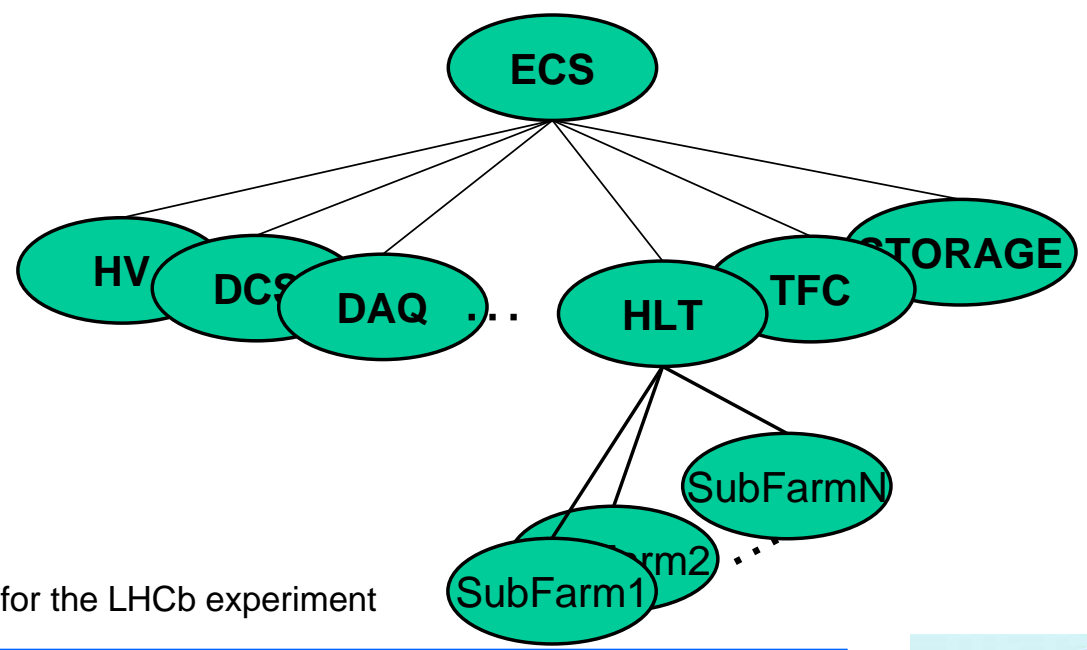




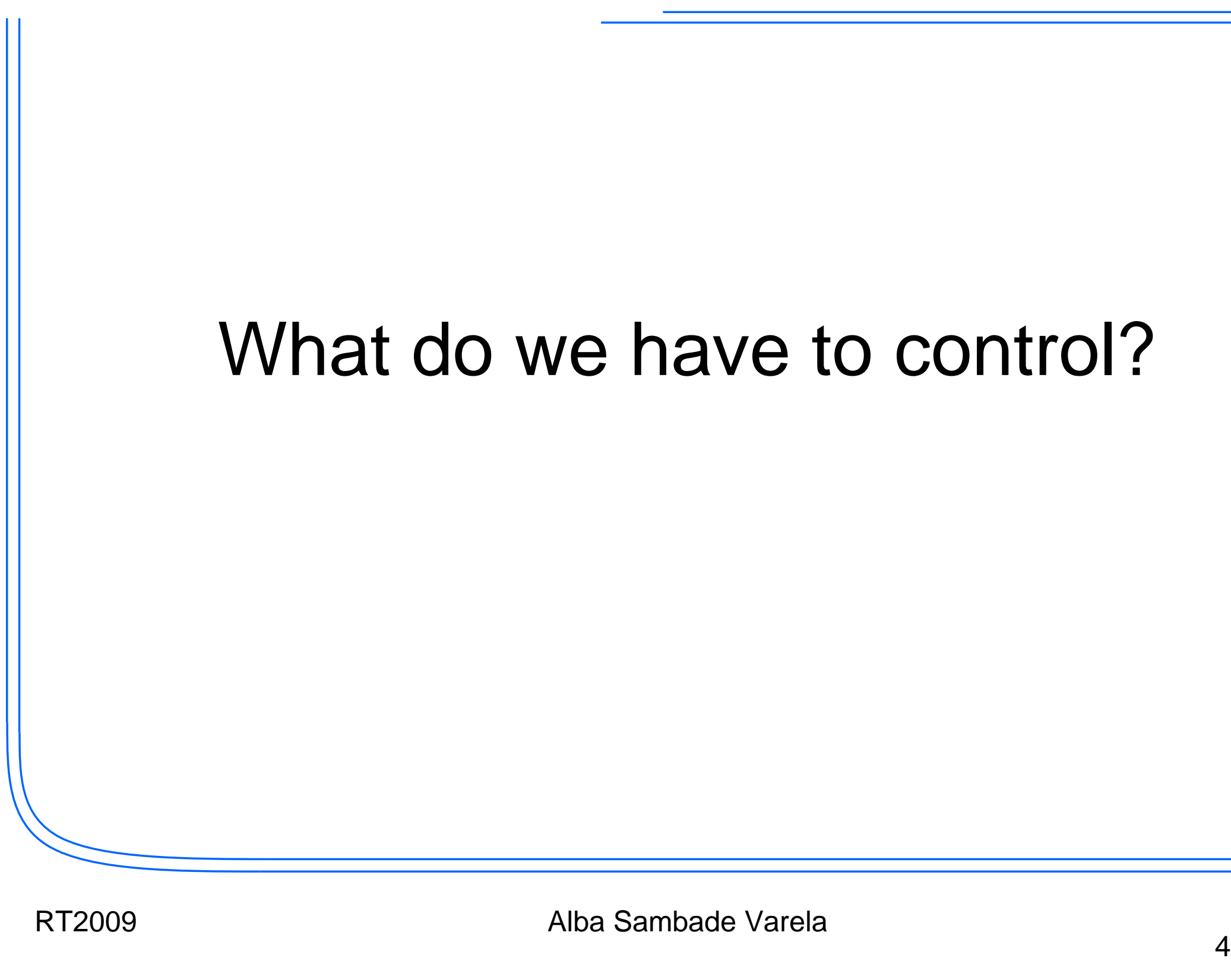




\section{High Level Trigger CPU Farm}

- Composed out of up to 2000 boxes (= nodes)

- grouped into 50 subfarms (50 racks)

- up to 40 nodes/subfarm

- 8 cores/node *

- 1 HLT algorithm running/core

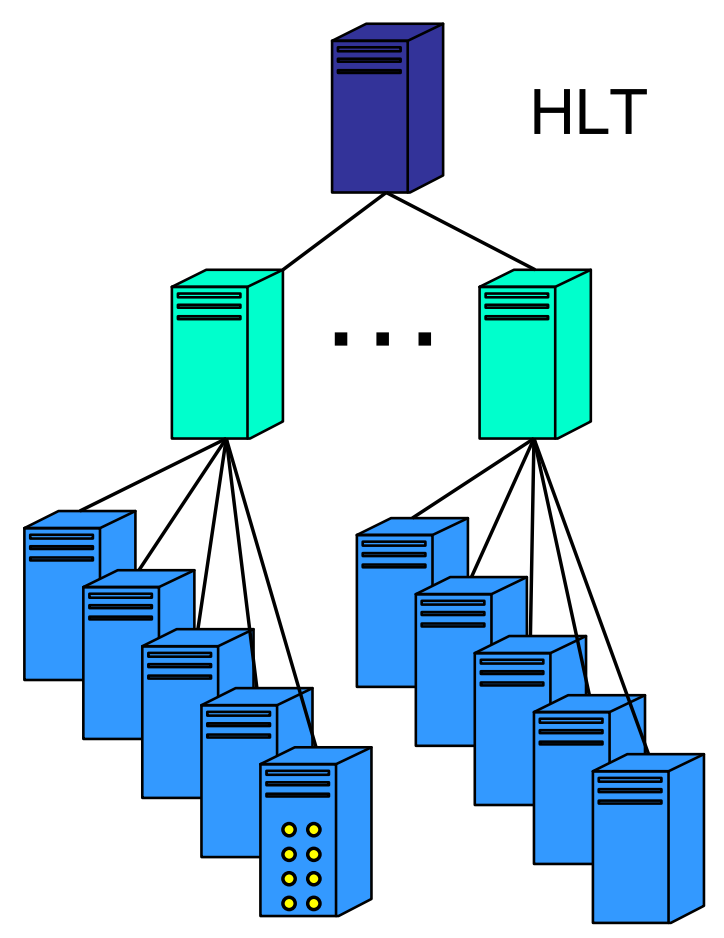




\section{HLT Top level control}

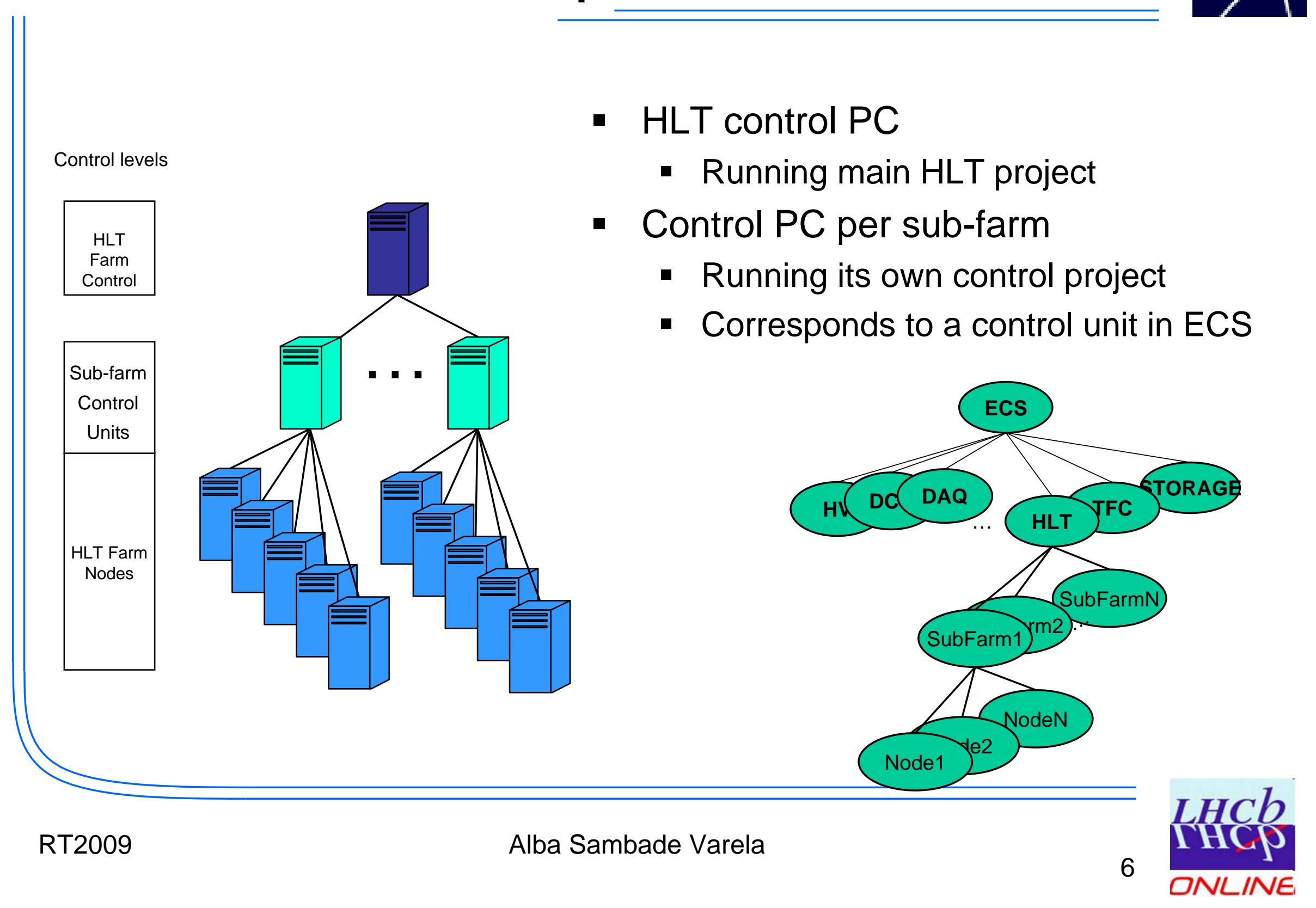




\section{HLT low level control}

- Logical aggregation

- Functional algorithms

- Division of HLT node by tasks

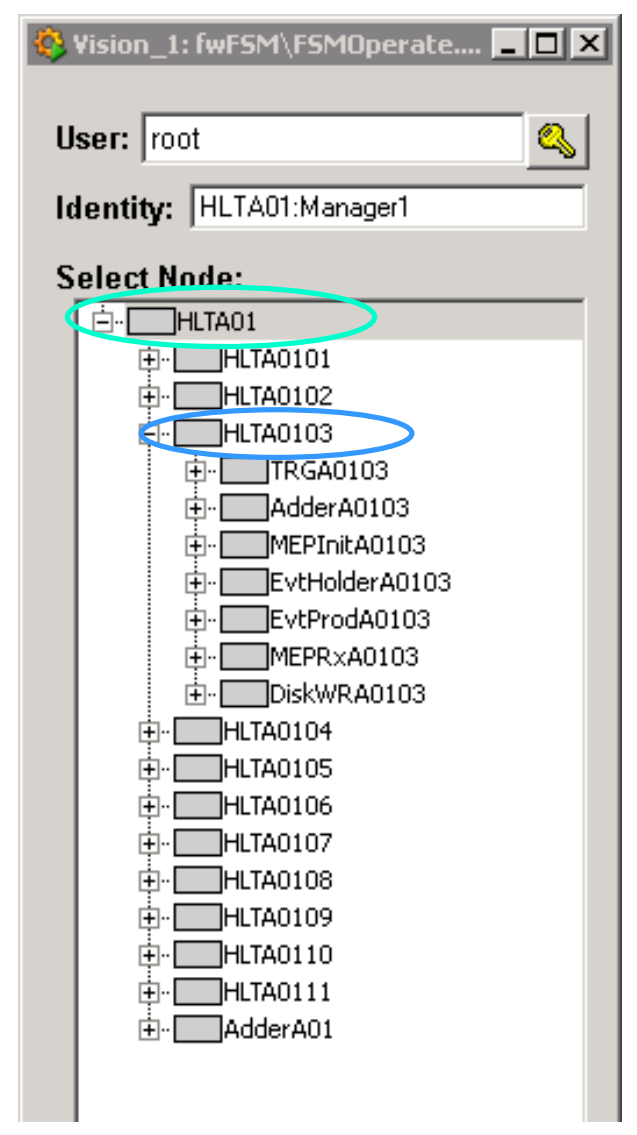




\section{Task architecture on HLT node}

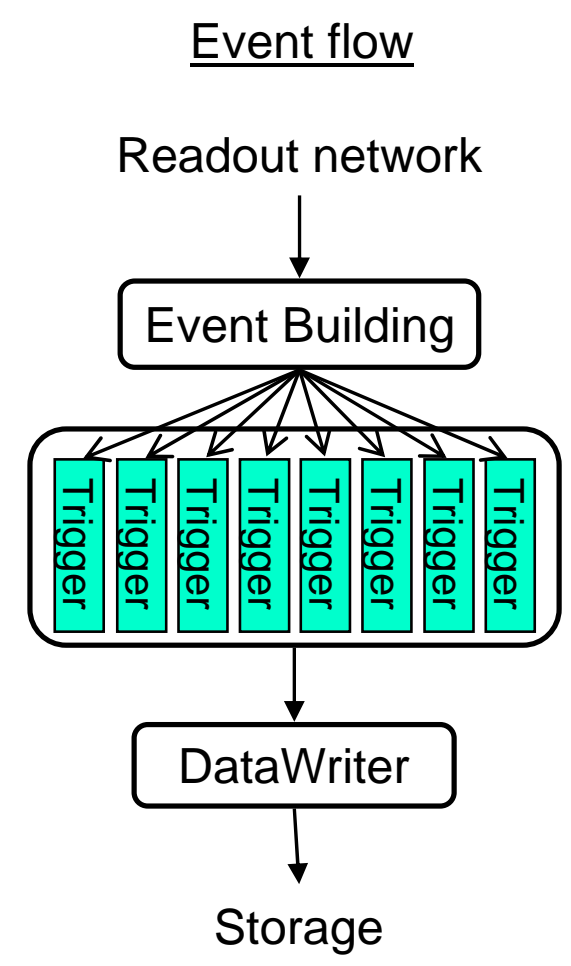

- Each node in the EFF runs one event-builder process.

- It distributes the assembled events to trigger processes.

- As many trigger-processes as there are CPU cores.

- Compute trigger decision and declare accepted events.

- Each node also runs one instance of the data-writer.

- Sends accepted events to Storage system.

- Algorithms implemented with GAUDI.

- GAUDI: data processing experiment independent framework.

- Same software used as for offline analysis. 


\section{HLT Tasks control}

- Algorithms are treated by the ECS as hardware devices.

- Integrated as Device Unit (DU)

- DU behavior modeled with Finite State Machines

- Common state diagram for all algorithms

- Transitions mapped to Gaudi transitions

- Command parameters

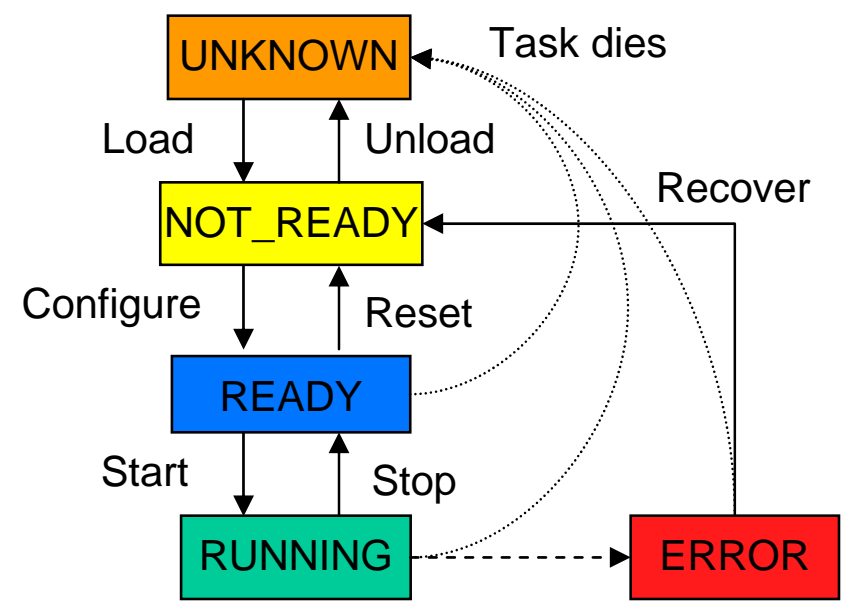




\section{Communication layer}

- Communication via Distributed Information Management System (DIM)

- Communication mechanism based on client-server paradigm

- Task behaves as a DIM server

- Publishes services

- Algorithm status

- Counters

- Receives commands

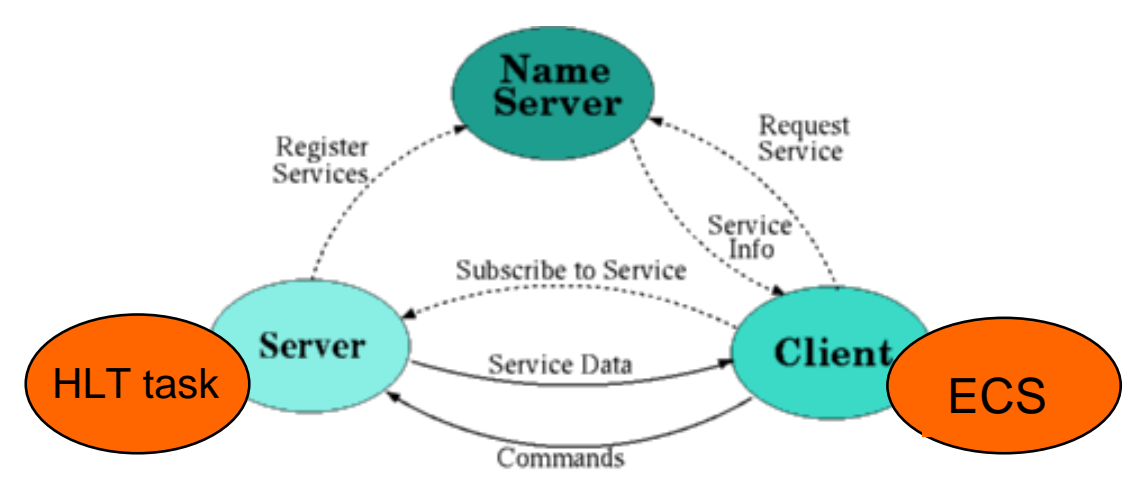

- Controls based on HLT naming convention

- Sub-farm (row and index)

- UTGID (task name) 


\section{Sub-Farm Control GUI}

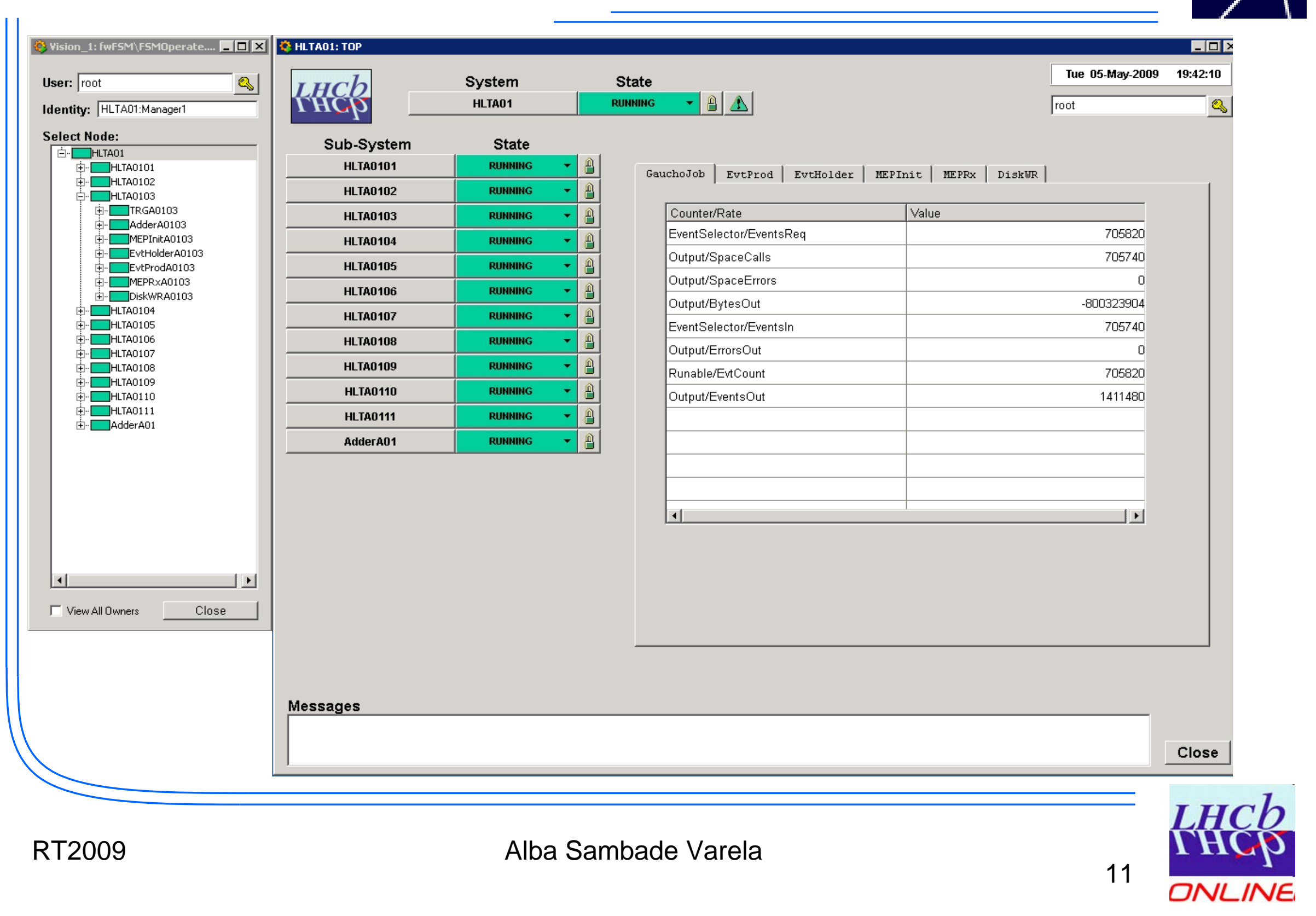




\section{Sub-farm control}

- Hierarchical ECS main control + dynamic allocation

- Easy to include new farms into global system

- Single sub-farm controls as framework package + base on naming convention

- Easy to duplicate farm control system.

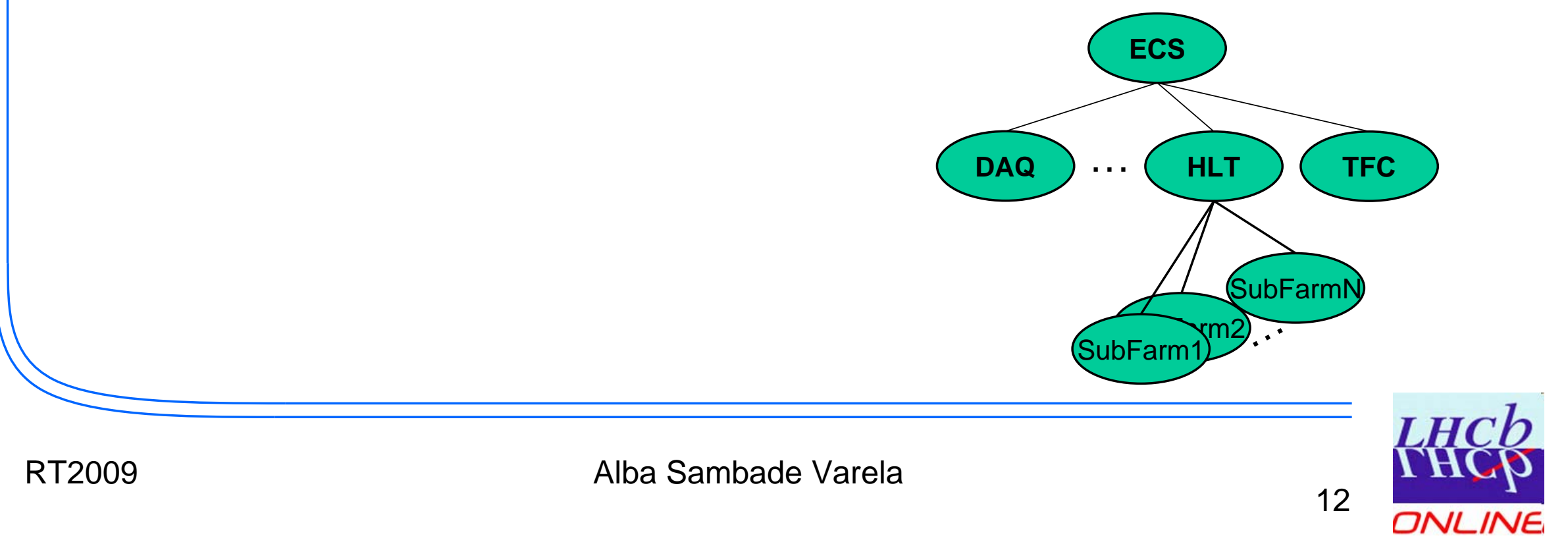




\section{Partitioning}

- Several sub-detectors (many teams)

- Different possible configurations (commissioning stage).

- Possibility to modify dynamically the readout components included to control.

- Different running modes (Physics, Calibration, Cosmics, etc).

- Run_type parameter sent with "Configure" command

- Pool of sub-farms $\rightarrow$ Dynamic allocation

- Different instances of run control running in parallel (readout partitions) 


\section{Farm pool controls}

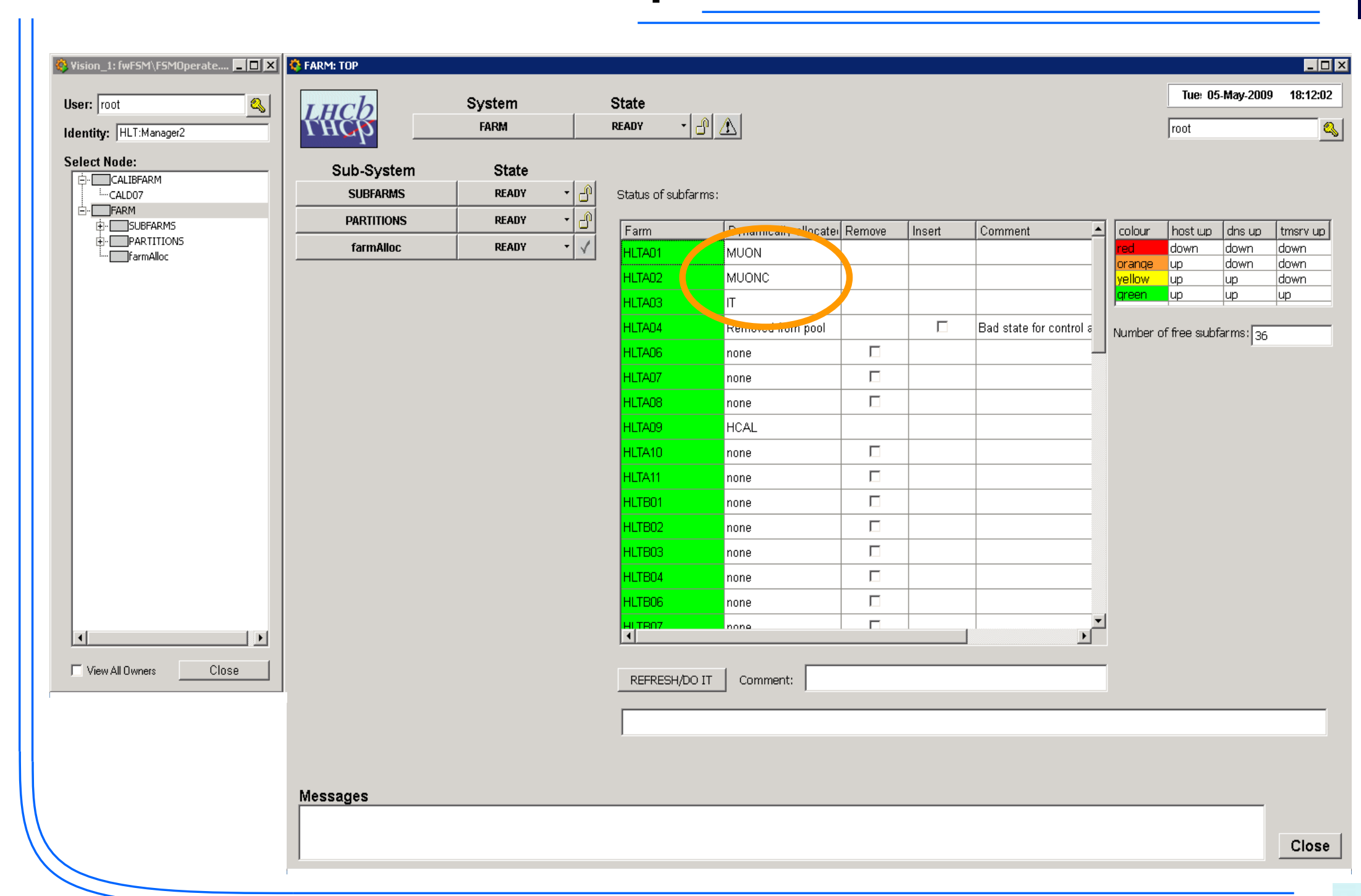




\section{Conclusions}

- HLT control completely defined and integrated into global LHCb control system.

- Implemented with same toolkit (PVSS \& FSM) used through the ECS.

- Keeps Homogeneity

- Fully configurable at real time.

- Automatic control of processes for the shift operator. 